\title{
Congestion Control in Wireless Sensor Networks Based on the Bird Flocking Behavior ${ }^{\star}$
}

\author{
Pavlos Antoniou ${ }^{1}$, Andreas Pitsillides ${ }^{1}$, \\ Andries Engelbrecht ${ }^{2}$, Tim Blackwell ${ }^{3}$, and Loizos Michael ${ }^{1}$ \\ 1 Department of Computer Science, University of Cyprus, Cyprus \\ 2 Department of Computer Science, University of Pretoria, South Africa \\ 3 Department of Computing, Goldsmiths College, University of London, UK
}

\begin{abstract}
Recently, performance controlled wireless sensor networks have attracted significant interest with the emergence of mission-critical applications (e.g. health monitoring). Performance control can be carried out by robust congestion control approaches that aim to keep the network operational under varying network conditions. In this study, swarm intelligence is successfully employed to combat congestion by mimicking the collective behavior of bird flocks, having the emerging global behavior of minimum congestion and routing of information flow to the sink, achieved collectively without explicitly programming them into individual nodes. This approach is simple to implement at the individual node, while its emergent collective behavior contributes to the common objectives. Performance evaluations reveal the energy efficiency of the proposed flock-based congestion control (Flock-CC) approach. Also, recent studies showed that Flock-CC is robust and self-adaptable, involving minimal information exchange and computational burden.
\end{abstract}

Keywords: Wireless Sensor Networks (WSNs), Congestion Control (CC).

\section{Introduction}

Typically, WSNs consist of small, cooperative devices (nodes) which may be constrained by computation capability, memory space, communication bandwidth and energy supply. The traffic load injected into the network can be unpredictable (e.g. due to event driven applications) and, in conjunction with variable wireless network conditions, may exceed available capacity at any point of the network resulting in congestion phenomena. Congestion causes energy waste, throughput reduction, increase in collisions and retransmissions at the MAC layer, increase of queueing delays and even information loss, leading to the deterioration of the offered quality and to the decrease of network lifetime.

The focal point of this study is to design a robust and self-adaptable congestion control $(C C)$ mechanism for WSNs. Inspiration is drawn from Swarm

\footnotetext{
* This work is supported in part by the GINSENG project funded by the FP7 under Grant No. ICT-224282 and the MiND2C project funded by the Research Promotion Foundation of Cyprus under Grant No. TPE/EPIKOI/0308(BE)/03.
} 
Intelligence (SI) 1], 2] which has been very successful in solving similar types of complex problems [3. However, the majority of SI-based network approaches like [3] target ad hoc networks which have fundamental differences from WSNs 4 . Our approach mimics the flocking behavior of birds, where packets are modeled as birds flying over a topological space, e.g. a sensor network. The main idea is to 'guide' packets to create flocks and 'fly' towards a global attractor (sink), whilst trying to avoid obstacles (congested regions). The direction of motion is influenced by (a) repulsion and attraction forces exercised by neighboring packets, as well as (b) the gravitational force in the direction of the sink. The flock-based congestion control (Flock-CC) approach provides sink direction discovery, congestion detection and traffic management. Flock-CC was initially proposed in [5] and with slight modifications in [6]. Both studies showed that the Flock-CC approach achieves low packet loss resulting in high packet delivery ratio (PDR) and thus reliability, low latency, and fault tolerance. Also it was found to outperform congestion-aware multi-path routing approaches in terms of PDR. This paper shows that Flock-CC also achieves low energy consumption.

The rest of this paper is organized as follows. Section 2 deals with the proposed approach. Section 3 presents performance evaluation results. Section 4 draws conclusions and proposes areas of future work.

\section{The Flock-Based Congestion Control (Flock-CC)}

The main concept of the Flock-CC approach [5, 6] is to 'guide' packets to form groups or flocks, and flow towards a global attractor (sink), whilst trying to avoid obstacles (congestion regions). In order to make moving packets behave like a flock, each packet interacts with neighboring packets on the basis of attraction and repulsion forces, and experiences a 'gravitational' force in the direction of the sink. These forces are synthesized in the decision making process of a packet, when a new hosting node is about to be chosen. In particular, packets are (a) attracted to neighboring packets located on nodes experiencing low queue loading, (b) repelled from neighboring packets located on nodes experiencing high wireless channel contention, (c) attracted to the sink by using biased preference to nodes located closer to the sink, whenever a new hosting node is about to be chosen, and (d) experience some perturbation that may help them to pick a random route.

\section{Congestion Detection and Sink Direction Discovery}

In wireless environments, packets may be dropped due to queue overflows and link layer failures (e.g. collisions) on each node. Based on these congestion symptoms and the queue state, a node loading indicator $p_{n}(k)$ 1 at node $n$ is devised:

$$
p_{n}(k)= \begin{cases}\frac{P_{n}^{\text {in }}(k)+q_{n}(k-1)-P_{n}^{\text {out }}(k)}{P_{n}^{\text {in }}(k)+q_{n}(k-1)}, & \text { if } P_{n}^{\text {in }, \text { out }}(k), q_{n}(k-1) \neq 0 ;, 0 \leq p_{n} \leq 1 \\ 0 & \text { otherwise. }\end{cases}
$$

\footnotetext{
${ }^{1}$ All quantities defined herein, are regularly sampled at discrete time intervals of $T$ seconds, are evaluated at each sensor node, and are broadcasted periodically (every $T$ seconds) to all neighboring nodes, using a dedicated control packet.
} 
where $P_{n}^{i n}(k)$ is the number of incoming packets, $P_{n}^{\text {out }}(k)$ is the number of successful outgoing packets at the end of the $k$-th period, and $q_{n}(k-1)$ is the queue size at the end of the $k-1$-th period at node $n$. More detailed information is given in [6]. When $p_{n}(k) \rightarrow 0$, both the number of packet drops at node $n$ is close to 0 and the queue is empty or nearly empty. On the other hand, as $p_{n}(k) \rightarrow 1$, node $n$ is considered congested due to either high number of packet drops, or high queue occupancy. Each node estimates the quality of the shared wireless channel (useful link service rate, $r_{n}(k)$ ), using information taken from the MAC protocol. This study considers CSMA-like MAC protocols (e.g. IEEE 802.11). The total number of all packet transmission attempts at node $n$ (during sampling period $k$ ) is denoted by $P_{n}^{o u t *}(k)$, where $P_{n}^{o u t *}(k)=P_{n}^{\text {out }}(k)+$ retransmits within that period. The useful link service rate at node $n$ is denoted by:

$$
r_{n}(k)=\left\{\begin{array}{ll}
1 & \text { if retransmits }=0 \text { and } P_{n}^{\text {out }}(k)=0 ; \\
\frac{P_{n}^{\text {out }}(k)}{P_{n}^{\text {out } *}(k)} \text { otherwise. }
\end{array}, \quad 0 \leq r_{n}(k) \leq 1 .\right.
$$

When $r_{n}(k) \rightarrow 1$, the channel is not congested and a large percentage of packets are successfully transmitted (few packet retransmissions are observed). As $r_{n}(k) \rightarrow 0$, the channel is congested and a small number of packets are successfully transmitted, often after a large number of retransmissions.

The direction of the sink can be deduced by the hop-distance variable, $h_{n}(k)$, indicating the number of hops between node $n$ and the sink at the $k$-th sampling period. Nodes located closer to the sink are expected to have smaller hop-distance values and should be chosen with higher probability as next hop hosting nodes.

\section{Traffic Management and Desirability}

Traffic management is performed on per packet basis in a hop-by-hop manner. Whenever a packet is about to be sent, the decision making process is invoked to determine the next hop node. Consider a network of $N$ nodes that are able to generate packets. Each hosting node evaluates the next hop node for each of its packets based on an $M$-dimensional desirability vector, $\vec{D}(k)$, where $M \leq N$, is the number of nodes located within the hosting node's transmission range. Each element, $D_{m}(k)$, of the vector $\vec{D}(k)$ represents the desirability for each node $m, m \in\{1, . ., M\}$. The desirability $D_{m}(k)$ for every node $m$ is evaluated once in each sampling period $k$ and is used for each packet sent within this period using:

$$
D_{m}(k)=\alpha \cdot r_{m}(k)+(1-\alpha) \cdot\left(1-p_{m}(k)\right),
$$

where the parameter $\alpha, 0 \leq \alpha \leq 1$, regulates the influence of parameters $r_{m}(k)$ and $p_{m}(k)$. In order to address global attractiveness to the sink, we allow packets to be forwarded even to nodes that are not closer to the sink, but we place some bias against such a choice, by discounting the desirability of such nodes over the nodes that are closer to the sink, using the discount factor $d_{i m}(t)$. In addition, we introduce randomness (that allows exploration, and perhaps identification of better solutions) in our model by introducing some noise in the desirability function. This perturbation is achieved by multiplying the desirability of a node by 
some coefficient drawn randomly from a Gaussian distribution with mean 1 and variance $v$. Let $g$ be a random variable that follows this probability distribution. Thus, we define the adjusted desirability of packet $i$ for node $m$ as:

$$
D_{i m}^{\prime}(t)=g \cdot d_{i m}(t) \cdot D_{m}(k) .
$$

Given the modified desirability function, our algorithm is as follows: For each packet $i$, choose node with the maximum adjusted desirability $m^{*}$, where: $m^{*}=\operatorname{argmax}_{m}\left\{\vec{D}_{i}^{\prime}(t)\right\}$. We consider nodes with only an equal or one less hop count to the sink than the hop count of the packet's current location. We set $d_{i m}(t)$ equal to $f=1$ for all nodes $m$ that are closer to the sink, and equal to some constant $e: 0 \leq e \leq 1$ for all nodes $m$ at equal distance from the sink. We also allow nodes of the latter category to be selected if the noise perturbation is sufficiently large to cover the bias against these nodes that is introduced by multiplying their desirability by $e$. The probability with which this bias $f-e$ is covered depends on the standard deviation $\sqrt{v}$ of the Gaussian distribution. It, then, makes sense to define $v$ not entirely independently of $f-e$, but as a linear function of it. We, thus, let $v=c \cdot(f-e)^{2}$ (and thus $\sqrt{v}$ is linear in $f-e$ ). Beyond the Gaussian approach the response threshold approach [8] was used in [5] for randomizing the attractiveness to the sink, achieving promising results.

The parameters $\alpha, e$, and $c$ are the most important control knobs of our system and are expected to influence its forwarding behavior. The parameter $\alpha$ regulates the influential roles of the node loading indicator $p_{m}(k)$, and the wireless channel loading $r_{m}(k)$ on the desirability function of Eq. 4. The parameter $e$ determines the bias against nodes at equal hop-count from the sink. The parameter $c$ determines, indirectly, the variation of the introduced noise.

\section{Performance Evaluation}

This section evaluates the performance of the Flock-CC approach through simulation studies conducted using the ns-2 network simulator. In accordance with [6], the optimal combination of the design parameters values achieving high packet delivery ratio (PDR) and low end-to-end delay (EED) was $a=0.4, e=0.6$ and $c=0.5$. In this paper, the energy tax metric is defined to evaluate the performance of the proposed approach. The energy tax calculates the average energy consumption per node per delivered packet. The evaluation topology consists of 200 homogeneous nodes deployed in a 2D lattice. The evaluation scenario involved the activation of 10 nodes located 7 hops away from the sink. Each active node was generating constant bit rate traffic at the rates of $25,35,45 \mathrm{pkts} / \mathrm{sec}$. These cases can be considered as slightly congested, congested, and heavily congested, respectively. The sampling period $T$ was set to 1 sec.

Fig. 1 illustrates the impact of $\alpha, e$ and $c$ on energy tax at $35 \mathrm{pkts} / \mathrm{sec}$. As can be seen, the lowest energy tax $(0.026 \mathrm{~mJ} /$ delivered packet $)$ was obtained for $\alpha=0.5, e=0.6$ and $c=0$ (dashed line of Fig. 1). Similarly, low energy tax levels were achieved for $0.2 \leq \alpha \leq 0.6,0 \leq e \leq 0.7$ and $0 \leq c \leq 0.25$. As stated before, parameter $e$ determines the bias against nodes at equal hop-count from 


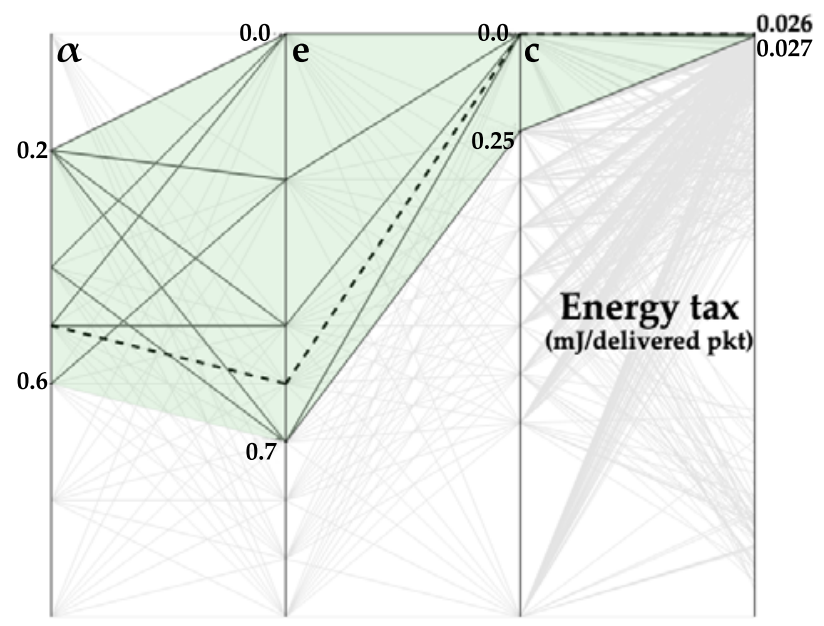

Fig. 1. Energy tax at 35pkts/sec

the sink. When $e=0$ packets can only move towards nodes located closer to sink (high flock coherence). On the other hand, as $e$ approaches to 1, packets are allowed to move towards nodes with equal hop-count with high probability (high traffic spreading). Perturbation is regulated by the parameter $c$. When $e$ and $c$ were close to 0 (i.e. high packet flock coherence and low perturbation), packets were moving on narrow paths to the sink resulting in low energy consumption per node. On the other hand, high packet spreading was observed for $e \rightarrow 1$ or $c \rightarrow 1$ causing transition loops of packets between uncongested nodes and, as a result, high energy consumption. Also, Fig. 1 shows that high energy consumption was observed for $\alpha>0.6$. The increase of $\alpha$, amplified the tendency of packets to move towards nodes experiencing low channel utilization (i.e. at the borders of the flock) in order to avoid collision hot spots occurring inside the network. However, packets were caught in loops between nodes experiencing low channel utilization, resulting in high energy consumption.

We implemented (a) a shortest-path congestion-unaware routing mechanism, and (b) a congestion-aware multi-path routing mechanism, according to which each node chooses the least congested next hop node in terms of queue length (among nodes involved in the shortest paths -if many- to the sink) to forward each packet. The Flock-CC approach $(\alpha=0.4, e=0.6, c=0.5)$ was compared with both approaches. This selection of values achieved the highest PDR [ 6] for the scenario under study, but also corresponds to low energy tax. Fig. 2(a) shows that Flock-CC paid $9.6 \%$ less energy tax than the congestion-unaware approach under low loads (25 pkts/sec), as well as $12.5 \%$ less under high loads (45 pkts/sec). Also, Flock-CC paid 4-10\% less energy tax compared with the congestion-aware approach. Fig. 2(b) shows that the superiority of Flock-CC is attributed to its ability to deliver larger amount of packets to the sink. 


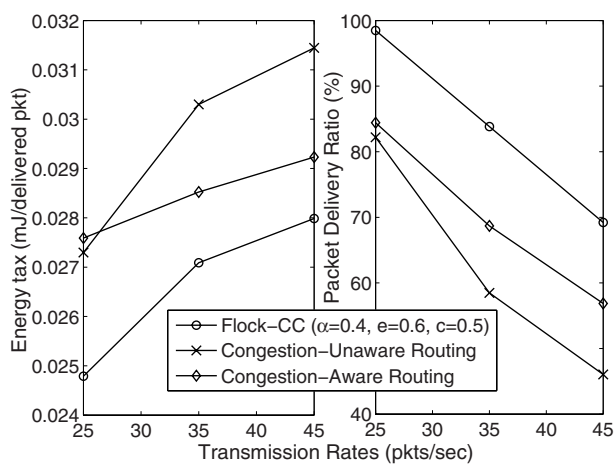

Fig. 2. Comparative experiments

\section{Conclusions and Future Work}

The aim of this study was to evaluate the Flock-CC mechanism from the perspective of energy consumption. Performance evaluations showed that under certain parameter values, the Flock-CC mechanism was able to both alleviate congestion [5, 6] and minimize energy tax. Also, Flock-CC outperformed congestion-aware and congestion-unaware routing approaches in terms of energy tax. The future work will provide more extensive comparative evaluations and a more thorough investigation of the influence of the various parameters. Also, the sampling period $T$ and its influence on performance, as well as the communication overhead from exchanging control packets are left for future work.

\section{References}

1. Bonabeau, E., Dorigo, M., Theraulaz, G.: Swarm Intelligence: From Natural to Artificial Systems. In: Proc. of Sciences of Complexity, Santa Fe Institute (1999)

2. Engelbrecht, A.: Fundamentals of Computational Swarm Intelligence. Wiley, Chichester (2005)

3. Di Caro, G., Ducatelle, F., Gambardella, L.M.: AntHocNet: An adaptive natureinspired algorithm for routing in mobile ad hoc networks. European Trans. on Telecom., Self-organization in Mobile Networking 16, 443-455 (2005)

4. Akyildiz, I.F., Su, W., Sankarasubramaniam, Y., Cayirci, E.: Wireless sensor networks: a survey. Computer Networks 38, 393-422 (2002)

5. Antoniou, P., Pitsillides, A., Blackwell, T., Engelbrecht, A.: Employing the Flocking Behavior of Birds for Controlling Congestion in Autonomous Decentralized Networks. In: Proc. of 2009 IEEE Congress on Evolutionary Computation (2009)

6. Antoniou, P., Pitsillides, A., Engelbrecht, A., Blackwell, T.: A Swarm Intelligence Congestion Control Approach for Autonomous Decentralized Communication Networks. In: Engelbrecht, A., Middendorf, M. (eds.) Applied Swarm Intelligence. SCI. Springer, Heidelberg (to appear)

7. Couzin, I.D., Krause, J., James, R., Ruxton, G., Franksz, N.: Collective Memory and Spatial Sorting in Animal Groups. Jrnl of Theor. Biology 218, 1-11 (2002)

8. Theraulaz, G., Bonabeau, E., Daneubourg, J.: Response Threshold Reinforcement and Division of Labour in Insect Societies, Royal Soc. of London, vol. 265, pp. 327-332. 